\title{
EXCEPTIONAL SINGULARITIES OF CODIMENSION ONE HOLOMORPHIC FOLIATIONS
}

\author{
Marco Brunella and Carlo Perrone
}

Abstract

We study some numerical properties of singularities of codimension one holomorphic foliations which can be analytically collapsed to one point. Some local and global dynamical consequences are deduced.

\section{Introduction}

Let $X$ be a complex manifold of dimension at least 3 , and let $\mathcal{F}$ be a codimension one holomorphic foliation on $X$. Denote by $\operatorname{Sing}(\mathcal{F})$ the singular set of $\mathcal{F}$, which is an analytic subset of $X$ of codimension at least 2, and denote by $\operatorname{Sing}_{*}(\mathcal{F})$ the union of the irreducible components of $\operatorname{Sing}(\mathcal{F})$ whose codimension is precisely 2 . This subset $\operatorname{Sing}_{*}(\mathcal{F})$ represents the most important part of $\operatorname{Sing}(\mathcal{F})$, for at least two (related) reasons. Firstly, according to Baum-Bott residue formula [B-B], [Suw], the cohomology class $c_{1}^{2}\left(N_{\mathcal{F}}\right)$ can be localized on $\operatorname{Sing}_{*}(\mathcal{F})$. Here $N_{\mathcal{F}}$ denotes the normal bundle of the foliation. Secondly, by Malgrange's singular Frobenius theorem [Mal] around each point of $\operatorname{Sing}(\mathcal{F}) \backslash \operatorname{Sing}_{*}(\mathcal{F})$ the foliation admits a holomorphic first integral, and so we may say that, in some sense, singularities outside $\operatorname{Sing}_{*}(\mathcal{F})$ are removable or negligible.

In this paper we shall study certain numerical properties of those connected components of $\operatorname{Sing}_{*}(\mathcal{F})$ which are exceptional subsets of $X$, that is which can be analytically collapsed to one point [Gra], [Pet]. The motivation comes from some problems tackled in $[\mathbf{B r} \mathbf{1}]$ and $[\mathbf{C}-\mathbf{L}]$.

In order to state our first and main result, let us recall that a line bundle $L$ on a compact Moishezon space $Z$ is said to be nef (resp. flat) if $\operatorname{deg}\left(\left.L\right|_{C}\right) \geq 0\left(\right.$ resp. $\left.\operatorname{deg}\left(\left.L\right|_{C}\right)=0\right)$ for every irreducible compact curve $C \subset Z$. These definitions make sense when $Z$ is an exceptional

2010 Mathematics Subject Classification. 32S65, 37F75, 14E15.

Key words. Holomorphic foliations, Baum-Bott residues, modifications.

Work partially supported by ANR-08-JCJC-0130-01. 
subset of a complex manifold, for such a subset is always a Moishezon space $[$ Pet, $\S 2.4]$.

Theorem 1.1. Let $\mathcal{F}$ be a codimension one foliation on a complex manifold $X$ of dimension at least 3 , and let $Z$ be a connected component of $\operatorname{Sing}_{*}(\mathcal{F})$ which is exceptional in $X$. Assume that $\left.N_{\mathcal{F}}\right|_{Z}$ is nef. Then:

(1) The Baum-Bott residue of each irreducible component of $Z$ is vanishing.

(2) $\left.N_{\mathcal{F}}\right|_{Z}$ is flat.

The proof of this result is based on Baum-Bott theory coupled with some vanishing theorems for line bundle cohomology $[\mathbf{G}-\mathbf{R}],[\mathbf{E}-\mathbf{V}]$. This will give a suitable division property of the foliation along $Z$, which in turn implies (1) and (2). As we shall see, however, part (1) holds under some slightly weaker assumption than part (2).

A first application of Theorem 1.1 concerns a conjecture stated in $[\mathbf{B r} \mathbf{1}]$ on the dynamics of codimension one foliations.

Theorem 1.2. Let $X$ be a complex projective manifold of dimension at least 3 and with $\operatorname{Pic}(X)=\mathbb{Z}$, and let $\mathcal{F}$ be a codimension one foliation on $X$. Then every leaf $L$ of $\mathcal{F}$ accumulates to $\operatorname{Sing}(\mathcal{F})$ :

$$
\bar{L} \cap \operatorname{Sing}(\mathcal{F}) \neq \emptyset .
$$

The link of this result with $[\mathbf{B r} \mathbf{1}]$ comes from the (easy) fact that the hypothesis $\operatorname{Pic}(X)=\mathbb{Z}$ implies that $N_{\mathcal{F}}$ is ample, hence Theorem 1.2 proves the conjecture of $[\mathbf{B r} \mathbf{1}]$ in the special case of projective manifolds with cyclic Picard group. The much more special case $X=\mathbb{C} P^{n}, n \geq 3$, was previously done in $[\mathbf{L i n}]$. The proof of Theorem 1.2 is based on Theorem 1.1 (in a slightly more general form) and a pseudoconvexity result from [Br2], which leads to exceptional singularities via Grauert's results $[\mathbf{G r a}]$, [Pet]. Theorem 1.1 is used in the following weak form: if $Z$ is an exceptional singularity, then $\left.N_{\mathcal{F}}\right|_{Z}$ cannot be ample.

A second application of Theorem 1.1 is relative to a local problem stated in $[\mathbf{C}-\mathbf{L}]$. Indeed, an exceptional singularity $Z$ is partially related to the work of Cerveau and Lins Neto, because by collapsing $Z$ to one point we get a foliation on a singular space $X_{0}$, with an isolated singularity $p_{0} \in X_{0}$, which is also an isolated singularity for the foliation (neglecting, as usual, the higher codimensional part of the singular set). However, we are not precisely in the setting considered in $[\mathbf{C - L}]$, because our projected foliation on $X_{0}$ is not necessarily generated by a holomorphic 1-form around $p_{0}$, as assumed everywhere in $[\mathbf{C}-\mathbf{L}]$.

We shall consider, in fact, only a rather particular case (which, anyway, is the case mentioned in [C-L, Problem 1]). Let $X_{0}$ denote the 
analytic space, of dimension 3 , defined by the equation $z_{1} z_{2}=z_{3} z_{4}$ in $\mathbb{C}^{4}$. It has an isolated singularity at the origin (which we shall call a simple double point). For sake of clarity, let us say that by a foliation on an open subset $V \subset X_{0}$ we just mean a foliation, in the ordinary sense, on $V^{\circ}=V \backslash\{0\}$; we do not impose any kind of condition at the origin. Cerveau and Lins Neto ask about the local structure of foliations on $X_{0}$.

Theorem 1.3. Let $\mathcal{F}$ be a codimension one foliation on a neighbourhood $V \subset X_{0}$ of 0 , and suppose that $\mathcal{F}$ is nonsingular on $V^{\circ}$. Then, up to restricting $V$, one of the following properties holds:

(1) $\mathcal{F}$ has a holomorphic first integral.

(2) Each leaf of $\mathcal{F}$ is a proper analytic subset of $V$ passing through the origin.

This result can be thought as a substitute to Malgrange's theorem [Mal] on singular threefolds having only simple double points as singularities. Remark that the general results of $[\mathbf{C}-\mathbf{L}]$ imply that property (1) holds provided that the foliation is generated by a holomorphic 1-form around 0, a sort of "Gorenstian" condition that, as we shall see, does not always hold. One could think that when property (2) holds then the foliation has a meromorphic first integral, but we shall see that it is not always the case. However, it is likely that, in that case, the foliation is topologically conjugate to one which admits a meromorphic first integral (compare with $[\mathbf{K l u}]$ ).

Besides Theorem 1.1, in the proof of Theorem 1.3 we shall use the remarkable fact that the singularity of $X_{0}$ can be resolved in two different ways by so-called small resolutions, related each other by a so-called flop.

The paper is divided into three sections, one for each theorem.

\section{Around Theorem 1.1}

We start by recalling some aspects of Baum-Bott residue formula $[\mathbf{B}-\mathbf{B}],[$ Suw $]$.

Let $\mathcal{F}$ be a codimension one foliation on a complex manifold $X$, of dimension $n \geq 2$. By definition, the datum of $\mathcal{F}$ is equivalent to specify a rank one saturated subsheaf

$$
N_{\mathcal{F}}^{\star} \subset \Omega_{X}^{1}
$$

which satisfies the Frobenius integrability condition. Locally, $N_{\mathcal{F}}^{\star}$ is generated by holomorphic 1 -forms $\omega_{j} \in \Omega_{X}^{1}\left(U_{j}\right)$, where $\left\{U_{j}\right\}$ is an open covering of $X$, such that

$$
\omega_{j} \wedge d \omega_{j}=0
$$


and

$$
\omega_{j}=g_{j k} \omega_{k} \quad \text { on } U_{j} \cap U_{k} .
$$

The functions $g_{j k}$ are nowhere vanishing, and the multiplicative cocycle $\left\{g_{j k}\right\}$ defines a line bundle $N_{\mathcal{F}}$, dual to $N_{\mathcal{F}}^{\star}$ and called normal bundle of $\mathcal{F}$. The saturatedness condition means that the zero set of every $\omega_{j}$ has codimension at least 2, i.e. $N_{\mathcal{F}}^{\star}$ is a line subbundle of $\Omega_{X}^{1}$ outside an analytic subset of codimension at least 2, called singular set of $\mathcal{F}$ and denoted by $\operatorname{Sing}(\mathcal{F})$. As in the Introduction, we will denote by $\operatorname{Sing}_{*}(\mathcal{F})$ the codimension 2 part of $\operatorname{Sing}(\mathcal{F})$. Moreover, we set

$$
X^{\circ}=X \backslash \operatorname{Sing}(\mathcal{F}) \quad \text { and } \quad X^{*}=X \backslash \operatorname{Sing}_{*}(\mathcal{F}) \text {. }
$$

In the nonsingular case, the Frobenius condition $\omega_{j} \wedge d \omega_{j}=0$ can be equivalently replaced by

$$
d \omega_{j}=\beta_{j} \wedge \omega_{j}
$$

for some $\beta_{j} \in \Omega_{X}^{1}\left(U_{j}\right)$ (at least if $U_{j}$ is Stein, which we will always assume without loss of generality). This is the classical division property of nonsingular integrable 1-forms, which is at the heart of Baum-Bott theory. Remark that, given $\omega_{j}$, such a $\beta_{j}$ is not unique, but it is uniquely defined up to addition of a 1 -form which vanishes on $\mathcal{F}$, i.e. up to a section of $N_{\mathcal{F}}^{\star}$ over $U_{j}$. On $U_{j} \cap U_{k}$ we then find

$$
\beta_{j} \wedge \omega_{j}=\frac{d g_{j k}}{g_{j k}} \wedge \omega_{j}+\beta_{k} \wedge \omega_{j}
$$

which precisely means that the 1 -form defined by

$$
\gamma_{j k}=\frac{d g_{j k}}{g_{j k}}-\beta_{j}+\beta_{k}
$$

is also a section of $N_{\mathcal{F}}^{\star}$, over $U_{j} \cap U_{k}$. Hence $\left\{\gamma_{j k}\right\}$ is a cocycle of 1 -forms vanishing on $\mathcal{F}$, and it corresponds to a cohomology class in $H^{1}\left(X, N_{\mathcal{F}}^{\star}\right)$. It is readily seen that this class is intrinsically defined by the foliation, i.e. it does not depend on the choices done so far.

In the singular case, the previous construction can be done on $X^{\circ}$, or even on $X^{*}$ : indeed, by a simple cohomological argument, on which we shall return, the division property holds even for integrable 1-forms whose zero set has codimension at least 3 [Mal]. Hence we get a well defined class

$$
B B_{\mathcal{F}} \in H^{1}\left(X^{*}, N_{\mathcal{F}}^{\star}\right)
$$

intrinsically associated to the foliation.

Actually, on $X^{\circ}$, or even on $X^{*}$ thanks to [Mal], the foliation is locally defined by closed 1 -forms $\omega_{j}$, hence we may choose $\beta_{j}=0$ and therefore the cocycle $\left\{\gamma_{j k}\right\}$ is nothing but than the logarithmic derivative 
of the cocycle $\left\{g_{j k}\right\}$ (which vanish on $\mathcal{F}$ because of the closedness of the generating 1-forms). In other words, the line bundle $N_{\mathcal{F}}$ has, as any line bundle on a complex manifold, a class in $H^{1}\left(X, \Omega_{X}^{1}\right)$ (logarithmic derivative); the integrability condition allows, on $X^{*}$, to lift this class to $H^{1}\left(X^{*}, N_{\mathcal{F}}^{\star}\right)$.

Baum-Bott formula is related to the extendibility of this class $B B_{\mathcal{F}}$ from $X^{*}$ to $X$. Take a point $p \in \operatorname{Sing}_{*}(\mathcal{F})$. We shall say that $B B_{\mathcal{F}}$ extends through $p$ if there exists a (small) ball $B_{p} \subset X$ centered at $p$ such that $B B_{\mathcal{F}}$ extends to a class in $H^{1}\left(X^{*} \cup B_{p}, N_{\mathcal{F}}^{\star}\right)$. Setting

$$
S\left(B_{p}\right)=\operatorname{Sing}_{*}(\mathcal{F}) \cap B_{p} \quad \text { and } \quad B_{p}^{*}=B_{p} \backslash S\left(B_{p}\right),
$$

and observing that the cohomology over $B_{p}$ is obviously trivial, we see (Mayer-Vietoris) that $B B_{\mathcal{F}}$ extends through $p$ if and only if

$$
\left.B B_{\mathcal{F}}\right|_{B_{p}^{*}}=0
$$

for some ball $B_{p}$ centered at $p$. Because $B B_{\mathcal{F}}$ is defined through the division property, the following fact should be evident.

Lemma 2.1. The class $B B_{\mathcal{F}}$ extends through $p$ if and only if there exists holomorphic 1-forms $\omega$ and $\beta$ on some neighbourhood of $p$ such that $\omega$ generates $N_{\mathcal{F}}^{\star}$ and $d \omega=\beta \wedge \omega$.

Proof: We can always take a generator $\omega$ of $N_{\mathcal{F}}^{\star}$ on some ball $B_{p}$. Then the vanishing of $\left.B B_{\mathcal{F}}\right|_{B_{p}^{*}}$ is clearly equivalent to the solvability of the equation $d \omega=\beta \wedge \omega$ on $B_{p}^{*}$. By Hartogs' theorem, a solution of this equation on $B_{p}^{*}$ holomorphically extends to $B_{p}$.

Remark that, because $S\left(B_{p}\right)$ has codimension 2 in $B_{p}$, the cohomology group $H^{1}\left(B_{p}^{*}, N_{\mathcal{F}}^{\star}\right)$ is infinite dimensional [B-S, Chapter 2], and so the vanishing of the class $\left.B B_{\mathcal{F}}\right|_{B_{p}^{*}}$ is certainly not a trivial condition. On the other hand, $H^{1}\left(B_{p} \backslash A, N_{\mathcal{F}}^{\star}\right)=0$ whenever $A$ is an analytic subset of codimension at least 3 [B-S, Chapter 2], and this is the reason for which the division property holds around points in $\operatorname{Sing}(\mathcal{F}) \backslash \operatorname{Sing}_{*}(\mathcal{F})[\mathbf{M a l}]$.

A way to measure the nontriviality of the class $B B_{\mathcal{F}}$ on $B_{p}^{*}$ consists in looking at its residue along $S\left(B_{p}\right)$. If we work with smooth sections of $N_{\mathcal{F}}^{\star}$, instead of holomorphic ones, the corresponding cohomology group is trivial, and so we can certainly find a smooth $(1,0)$-form $\beta \in A^{1,0}\left(B_{p}^{*}\right)$ such that $d \omega=\beta \wedge \omega\left(\omega\right.$ being a generator of $\left.N_{\mathcal{F}}^{\star}\right)$. The smooth 3-form (of mixed type $(3,0)+(2,1)$ )

$$
\frac{1}{(2 \pi \mathrm{i})^{2}} \beta \wedge d \beta
$$


is closed, and it has a De Rham cohomology class in $H^{3}\left(B_{p}^{*}, \mathbb{C}\right)$, which does not depend on the choice of $\omega$ and $\beta$.

Take now an irreducible component $Y$ of $\operatorname{Sing}_{*}(\mathcal{F})$. Take a generic point $p \in Y$, i.e. a point where $Y$ is smooth and disjoint from the other singular components. Take $B_{p}$ sufficiently small, so that $S\left(B_{p}\right)$ is a codimension 2 subball of $B_{p}$. Then the above De Rham class can be integrated over an oriented 3 -sphere $L_{p} \subset B_{p}^{*}$ positively linked with $S\left(B_{p}\right)$ :

$$
B B(\mathcal{F}, Y)=\frac{1}{(2 \pi \mathrm{i})^{2}} \int_{L_{p}} \beta \wedge d \beta .
$$

This complex number is called Baum-Bott residue of $\mathcal{F}$ along $Y$. By a connectedness argument, it does not depend on the choice of the generic point $p \in Y$.

Remark that $B B(\mathcal{F}, Y)=0$ when $B B_{\mathcal{F}}$ extends through $p$ (it is not difficult to see that this condition also does not depend on the choice of the generic $p \in Y$ ), hence the Baum-Bott residue represents the first obstruction for the extension of $B B_{\mathcal{F}}$ from $X^{*}$ to $X$. Generally speaking, however, there are also "higher order" obstructions.

It is now an easy matter to get Baum-Bott formula. To state it, let us recall that every irreducible component $Y$ of $\operatorname{Sing}_{*}(\mathcal{F})$ has a class $[Y] \in$ $H^{4}(X, \mathbb{C})$ (conveniently defined via the integration current over $Y$ ).

Theorem 2.1 ([B-B] $)$.

$$
c_{1}^{2}\left(N_{\mathcal{F}}\right)=\sum_{Y} B B(\mathcal{F}, Y)[Y]
$$

where the sum is done over all irreducible components of $\operatorname{Sing}_{*}(\mathcal{F})$.

Proof: We sketch a proof because our definition of $B B(\mathcal{F}, Y)$ is hard to find explicitely in [B-B] or [Suw].

We cover $X$ by open sets $U_{j}$ where the foliation is defined by holomorphic 1-forms $\omega_{j}$, with $\omega_{j}=g_{j k} \omega_{k}$. We may find smooth (1,0)-forms $\beta_{j}$ on $U_{j}^{*}=U_{j} \cap X^{*}$ such that $d \omega_{j}=\beta_{j} \wedge \omega_{j}$. We fix a small neighbourhood $V$ of $\operatorname{Sing}_{*}(\mathcal{F})$ and we regularize each $\beta_{j}$ on $V$, i.e. we choose a smooth $(1,0)$-form $\widetilde{\beta}_{j}$ on $U_{j}$ coinciding with $\beta_{j}$ outside of $U_{j} \cap V$. Then the smooth $(1,0)$-forms $\widetilde{\gamma}_{j k}=\frac{d g_{j k}}{g_{j k}}-\widetilde{\beta}_{j}+\widetilde{\beta}_{k}$ vanish on $\mathcal{F}$ outside of $V$. This cocycle can be trivialized: $\widetilde{\gamma}_{j k}=\widetilde{\gamma}_{j}-\widetilde{\gamma}_{k}$, where $\widetilde{\gamma}_{j}$ is a smooth $(1,0)$-form on $U_{j}$ vanishing on $\mathcal{F}$ outside of $U_{j} \cap V$. Finally, after setting $\widehat{\beta}_{j}=\widetilde{\beta}_{j}+\widetilde{\gamma}_{j}$, we find

$$
\frac{d g_{j k}}{g_{j k}}=\widehat{\beta}_{j}-\widehat{\beta}_{k} .
$$

Remark that we still have $d \omega_{j}=\widehat{\beta}_{j} \wedge \omega_{j}$ outside of $U_{j} \cap V$. 
The globally defined closed 2-form (of mixed type $(2,0)+(1,1)$ )

$$
\Omega=\frac{1}{2 \pi \mathrm{i}} d \widehat{\beta}_{j}
$$

represents, in the De Rham sense, the Chern class of $N_{\mathcal{F}}$, and so its square $\Omega \wedge \Omega$ represents $c_{1}^{2}\left(N_{\mathcal{F}}\right)$. Outside of $V$, by differentiating the identity $d \omega_{j}=\widehat{\beta}_{j} \wedge \omega_{j}$ we get $\Omega \wedge \omega_{j}=0$, from which it follows $\Omega \wedge \Omega=0$, that is

$$
\operatorname{Supp}(\Omega \wedge \Omega) \subset \bar{V}
$$

If $T \subset X$ is a 2-ball intersecting transversely $\operatorname{Sing}_{*}(\mathcal{F})$ at a single point $p \in Y$, with $V \cap T \Subset T$, then the integral of $\Omega \wedge \Omega$ on $T$ is equal to $B B(\mathcal{F}, Y)$, by Stokes formula, i.e. residue calculus. This means that the 4 -form $\Omega \wedge \Omega$ is cohomologous, as a current, to the integration current over $\sum_{Y} B B(\mathcal{F}, Y)[Y]$.

We can now start the proof of Theorem 1.1. From now on, we shall suppose that $n=\operatorname{dim} X \geq 3$.

Let $Z$ be a connected component of $\operatorname{Sing}_{*}(\mathcal{F})$, with irreducible components $Z_{j}, j=1, \ldots, \ell$. Suppose that $Z$ is contained in an exceptional subset $W \subset X$. Recall [Gra], [Pet, $\S 2$ ] that this means that $W$ is a compact connected analytic subset of $X$ for which there exists a normal analytic space $X_{0}$ and a proper holomorphic map

$$
\pi: X \longrightarrow X_{0}
$$

such that $\pi(W)$ is a single point $p$ of $X_{0}$ (usually singular) and the restriction $\left.\pi\right|_{X \backslash W}$ is a biholomorphism onto $X_{0} \backslash\{p\}$.

Remark that we do not require the equality $W=Z$ : the exceptional $W$ may be larger than $Z$, and it may have irreducible components of any dimension (between 1 and $n-1$ ), not necessarily singular for the foliation. However, we shall always require, even if not explicitely stated, that for some neighbourhood $U$ of $W$ we have

$$
\operatorname{Sing}_{*}(\mathcal{F}) \cap U \subset W
$$

(this condition is automatically satisfied if $W=Z$ ).

For any irreducible component $Z_{j}$ of $Z$, denote by $W\left(Z_{j}\right)$ the union of the irreducible components of $W$ which contain $Z_{j}$. Of course, we have only two possibilities: either $W\left(Z_{j}\right)=Z_{j}$, or $W\left(Z_{j}\right)$ is a union of hypersurfaces containing $Z_{j}$. We shall say that $Z_{j}$ is a fat component if the former case occurs. Given any point $p \in Z$, we shall say that $p$ is a fat point if every irreducible component of $Z$ through $p$ is fat. In particular, this holds when the codimension of $W$ at $p$ is at least 2 (but 
not conversely: $W$ may have an irreducible component of codimension one through $p$ which contains no component of $Z$ ).

The following statement implies part (1) of Theorem 1.1.

Proposition 2.1. Let $Z$ be a connected component of $\operatorname{Sing}_{*}(\mathcal{F})$ which is contained in an exceptional subset $W \subset X$. Assume that $\left.N_{\mathcal{F}}\right|_{W}$ is nef. Then, for every fat point $p \in Z$, the class $B B_{\mathcal{F}}$ extends through $p$. In particular, for every fat irreducible component $Z_{j}$ of $Z$, we have

$$
B B\left(\mathcal{F}, Z_{j}\right)=0 .
$$

Proof: The fact that $W$ is exceptional in $X$ guarantees that there exists a (arbitrarily small) strongly pseudoconvex neighbourhood $U \subset X$ of $W$, such that $W$ is the maximal positive dimensional analytic subset of $U$ (this is the easy part of [Gra], which proves that the converse also holds). We choose $U$ so that $\operatorname{Sing}_{*}(\mathcal{F}) \cap U \subset W$, that is $U \backslash W \subset X^{*}$. Hence the class $B B_{\mathcal{F}}$ restricts to a class

$$
\left.B B_{\mathcal{F}}\right|_{U \backslash W} \in H^{1}\left(U \backslash W, N_{\mathcal{F}}^{\star}\right) .
$$

We will show that this class extends to $U$. This implies the desired conclusion, because around each fat point any irreducible components of $Z$ is also an irreducible component of $W$. It is here worth observing that, by the usual codimension 3 argument, the class $\left.B B_{\mathcal{F}}\right|_{B_{p}^{*}}$ extends to $B_{p}$ if and only if it extends through generic points of $S\left(B_{p}\right)$.

We shall use the following vanishing theorem, due to Grauert-Riemenschneider $[\mathbf{G}-\mathbf{R}]$ and Kawamata-Viehweg $[\mathbf{E}-\mathbf{V}]$ : if $L$ is a line bundle on $U$ such that $\left.L\right|_{W}$ is nef, then $H^{k}\left(U, K_{X} \otimes L\right)=0$ for every $k \geq 1$. Actually, this is proved in [G-R] under the slightly stronger hypothesis that $L$ admits a smooth hermitian metric with semipositive curvature. The proof is by reduction, via an algebraization theorem of Artin, to an analogous vanishing theorem for so-called "quasi-positive" line bundles on projective manifolds, still due to $[\mathbf{G}-\mathbf{R}]$. This last result has been generalized by Kawamata and Viehweg to the case of nef and big line bundles $[\mathbf{E}-\mathbf{V}, \S 5]$. Then the same reduction procedure of GrauertRiemenschneider gives the statement that we are using.

We apply this vanishing theorem to $L=N_{\mathcal{F}}$ and $k=n-2$ (it is here that the assumption $n \geq 3$ is essentially used). Then, by Serre's duality [B-S, Chapter 7], we obtain

$$
H_{\text {cpt }}^{2}\left(U, N_{\mathcal{F}}^{\star}\right)=0
$$

(cohomology with compact support). This vanishing holds also for any other smaller strongly pseudoconvex neighbourhood $U^{\prime} \subset U$ of $W$, hence, 
as in [B-S, Chapter 1], we find

$$
H_{W}^{2}\left(U, N_{\mathcal{F}}^{\star}\right)=0
$$

(cohomology with support in $W$ ). Consider now the exact sequence

$$
H^{1}\left(U, N_{\mathcal{F}}^{\star}\right) \rightarrow H^{1}\left(U \backslash W, N_{\mathcal{F}}^{\star}\right) \rightarrow H_{W}^{2}\left(U, N_{\mathcal{F}}^{\star}\right) .
$$

From the vanishing of the last term we infer that any class in the group $H^{1}\left(U \backslash W, N_{\mathcal{F}}^{\star}\right)$ extends to $H^{1}\left(U, N_{\mathcal{F}}^{\star}\right)$.

Remark 2.1. The referee has suggested the following reformulation of the previous proof. Instead of working on the family of strongly pseudoconvex neighbourhoods $U^{\prime} \supset W$, we can consider the formal completion of $X$ along $W$, denoted by $\widehat{X}_{W}$. Then, by a suitable version of Kawamata-Viehweg theorem for formal spaces, one can obtain the vanishing of the group $H^{n-2}\left(\widehat{X}_{W}, K_{X} \otimes N_{\mathcal{F}}\right)$, that is, by Serre's duality, of $H_{W}^{2}\left(X, N_{\mathcal{F}}^{\star}\right)\left(=H_{W}^{2}\left(U, N_{\mathcal{F}}^{\star}\right)\right)$. The advantage of this approach is that it gives directly the vanishing that we are looking for, without passing through the groups $H_{\mathrm{cpt}}^{2}\left(U^{\prime}, N_{\mathcal{F}}^{\star}\right)$. The inconvenient is that it needs a vanishing theorem of Kawamata-Viehweg type which is difficult to find in the literature. On the other hand, let us observe that, by an easy variation on Lemma 2.1, the vanishing of $H_{\mathrm{cpt}}^{2}\left(U, N_{\mathcal{F}}^{\star}\right)$ for some $U$ is already sufficient to extend the class to $U$.

With a stronger assumption on $W$, we now can prove also part (2) of Theorem 1.1.

Proposition 2.2. Let $Z$ be a connected component of $\operatorname{Sing}_{*}(\mathcal{F})$ which is contained in an exceptional subset $W \subset X$ of codimension 2 (i.e., every irreducible component of $W$ has codimension at least 2). Assume that $\left.N_{\mathcal{F}}\right|_{W}$ is nef. Then $\left.N_{\mathcal{F}}\right|_{Z}$ is flat.

Proof: The codimensional hypothesis on $W$ ensures that every $p \in Z$ is a fat point. By the previous proposition, there exists a covering $\left\{U_{j}\right\}$ of a neighbourhood of $Z$ and holomorphic 1-forms $\omega_{j}, \beta_{j} \in \Omega_{X}^{1}\left(U_{j}\right)$ such that $\omega_{j}$ generates $N_{\mathcal{F}}^{\star}, d \omega_{j}=\beta_{j} \wedge \omega_{j}, \omega_{j}=g_{j k} \omega_{k}$. As usual, we find on $U_{j} \cap U_{k}$ the identity

$$
\frac{d g_{j k}}{g_{j k}} \wedge \omega_{j}=\left(\beta_{j}-\beta_{k}\right) \wedge \omega_{j}
$$

which means that $\frac{d g_{j k}}{g_{j k}}-\beta_{j}+\beta_{k}$ is a section of $N_{\mathcal{F}}^{\star}$ over $U_{j} \cap U_{k}$, i.e. it is proportional to the generator $\omega_{j}$. In particular, and because $\omega_{j}$ vanishes identically on $Z \subset \operatorname{Sing}(\mathcal{F})$, we find that

$$
\left.\frac{d g_{j k}}{g_{j k}}\right|_{Z}=\left.\beta_{j}\right|_{Z}-\left.\beta_{k}\right|_{Z}
$$


Now, by this relation the 2 -form $\Omega \in \Omega_{Z}^{2}(Z)$ locally defined by $\left.\frac{1}{2 \pi \mathrm{i}} d \beta_{j}\right|_{Z}$ represents the Chern class of $\left.N_{\mathcal{F}}\right|_{Z}$. Because it is holomorphic, its integral over a compact complex curve in $Z$ is vanishing. This means that $\left.N_{\mathcal{F}}\right|_{Z}$ is flat.

The argument used in the previous proof is nothing but than a "singular" version of Bott vanishing principle (which, by the way, is at the origin of Baum-Bott formula), which says that $N_{\mathcal{F}}$ is flat along the nonsingular leaves of $\mathcal{F}$. We just showed that such a flatness holds also along singular leaves (i.e. locally analytic subsets invariant by $\mathcal{F}$ but possibly passing through $\operatorname{Sing}(\mathcal{F})$, or even contained in it), provided that at each point of such a singular leaf the division property is satisfied.

One could ask if, under the hypotheses of Proposition 2.2, one has the flatness of $N_{\mathcal{F}}$ on $W$, and not only $Z$. The answer is negative, because $W$ could contain a "supplementary" irreducible component which is not singular for $\mathcal{F}$ and not even invariant by $\mathcal{F}$, thus not subject to Bott vanishing principle (cf. the examples at the end of the paper).

\section{Around Theorem 1.2}

Let us firstly recall a result from $[\mathbf{B r 2}]$.

Proposition 3.1 ([Br2, Proposition 3.1]). Let X be a complex projective manifold and let $\mathcal{F}$ be a codimension one foliation on $X$ whose normal bundle $N_{\mathcal{F}}$ is ample. Let $\mathcal{M} \subset X$ be a compact subset invariant by $\mathcal{F}$ and disjoint from $\operatorname{Sing}(\mathcal{F})$. Then $X \backslash \mathcal{M}$ is strongly pseudoconvex.

The ampleness assumption in that result is always satisfied whenever the Picard group is cyclic:

Lemma 3.1. Let $X$ be a complex projective manifold with $\operatorname{Pic}(X)=\mathbb{Z}$. Then every codimension one foliation on $X$ has ample normal bundle.

Proof: The normal bundle cannot be trivial, otherwise the foliation would be globally generated by a holomorphic 1-form, against the hypothesis $\operatorname{Pic}(X)=\mathbb{Z}$ (which, in the projective case, implies that $\Omega_{X}^{1}(X)=$ $\{0\}$ ). Hence, either $N_{\mathcal{F}}$ or $N_{\mathcal{F}}^{\star}$ is ample. The latter possibility is however excluded by an inequality of Bogomolov-Castelnuovo-De Franchis [Rei], which states, more specifically, that the conormal bundle of a codimension one foliation has Iitaka-Kodaira dimension at most 1.

The proof of Theorem 1.2 is by contradiction. Suppose that, for some leaf $L$ of $\mathcal{F}$, we have $\bar{L} \cap \operatorname{Sing}(\mathcal{F})=\emptyset$, so that $\mathcal{M}=\bar{L}$ is compact, 
invariant by the foliation and disjoint from its singularities. By Proposition $3.1, X \backslash \mathcal{M}$ is strongly pseudoconvex, i.e. there exists a smooth function

$$
\psi: X \backslash \mathcal{M} \longrightarrow \mathbb{R}
$$

such that $\psi(p) \rightarrow+\infty$ as $p \rightarrow \mathcal{M}$ and $d d^{c} \psi>0$ outside a compact set $K \subset X \backslash \mathcal{M}$. According to Grauert [Gra], [Pet, §2], $X \backslash \mathcal{M}$ contains a maximal positive dimensional compact analytic subset $W^{\prime}$, all of whose connected components are exceptional subsets, and by contracting each such component to a point we get a (singular) Stein space (the so-called Remmert reduction).

The assumption $\operatorname{Pic}(X)=\mathbb{Z}$ implies that every irreducible component of $W^{\prime}$ has codimension at least 2: indeed, every hypersurface in $X$ is ample, hence some multiple of it moves in a nontrivial family, and hence it cannot be inside an exceptional subset.

Consider now the codimension 2 singular set $\operatorname{Sing}_{*}(\mathcal{F})$. It is compact and contained in $X \backslash \mathcal{M}$, hence in $W^{\prime}$. Any connected component $Z$ is contained in a connected component $W$ of $W^{\prime}$. Of course, $\left.N_{\mathcal{F}}\right|_{W}$ is nef, and even ample, hence we may apply Proposition 2.2 and conclude that $\left.N_{\mathcal{F}}\right|_{Z}$ is flat. This contradicts the ampleness, unless $\operatorname{Sing}_{*}(\mathcal{F})=\emptyset$. In this last case, however, from Baum-Bott formula we get

$$
c_{1}^{2}\left(N_{\mathcal{F}}\right)=0
$$

which is again in contradiction with the ampleness. This completes the proof of Theorem 1.2.

Note that instead of using Proposition 2.2 we may use the weaker Proposition 2.1, which still gives $B B(\mathcal{F}, Y)=0$ for every irreducible component of the singular set, and consequently $c_{1}^{2}\left(N_{\mathcal{F}}\right)=0$ again. This could be useful in trying to prove the conjecture of $[\mathbf{B r 1} \mathbf{1}$ in full generality, i.e. under the sole assumption that $N_{\mathcal{F}}$ is ample and no assumption on the Picard group. In that case, we are faced with the problem that $W^{\prime}$ may contain some codimension one irreducible component. We should need a stronger conclusion in Proposition 2.1, ensuring the vanishing (or negativity...) of $B B(\mathcal{F}, Y)$ even for irreducible components $Y$ which are not fat.

Remark 3.1. It is not difficult to see (using [Mal]) that the above Proposition 3.1 from $[\mathbf{B r 2}]$ holds under the weaker hypothesis that $\mathcal{M}$ is disjoint from $\operatorname{Sing}_{*}(\mathcal{F})$, instead of $\operatorname{Sing}(\mathcal{F})$. Therefore, the conclusion of Theorem 1.2 can be improved as

$$
\bar{L} \cap \operatorname{Sing}_{*}(\mathcal{F}) \neq \emptyset .
$$




\section{Around Theorem 1.3}

In this section we shall work on a complex manifold $X$ of dimension 3 (a threefold). A compact connected component $Z$ of $\operatorname{Sing}_{*}(\mathcal{F})$ is therefore a (connected) union of irreducible compact curves $C_{1}, \ldots, C_{\ell}$. To say that $Z$ is contained in a codimension 2 exceptional subset $W$ is the same as to say that $Z$ itself is exceptional in $X$ (once a time, this follows from [Gra]: using the fact that $\overline{W \backslash Z}$ and $Z$ intersect each other only at isolated points, it is not difficult to construct a fundamental system of strongly pseudoconvex neighbourhoods of $Z$ starting from an analogous fundamental system of $W$ ). Theorem 1.1 says that, if $Z$ is exceptional, then the conditions $\left\{\left.\operatorname{deg} N_{\mathcal{F}}\right|_{C_{j}} \geq 0\right.$ for every $\left.j\right\}$ actually imply $\left\{\left.\operatorname{deg} N_{\mathcal{F}}\right|_{C_{j}}=0\right.$ for every $\left.j\right\}$. In particular, if $Z=C$ is irreducible, then we necessarily have

$$
\left.\operatorname{deg} N_{\mathcal{F}}\right|_{C} \leq 0
$$

By collapsing $Z$ to one point we get an analytic space $X_{0}$ with an isolated singularity $p_{0}$. The collapsing map $\pi: X \rightarrow X_{0}$ is said to be a small resolution of $p_{0}$, because its exceptional locus (where it fails to be an isomorphism) has codimension 2. Of course, the existence of a small resolution for an isolated singular point is a rather strong property. The singularity $p_{0}$ is said to be rational if, for every sufficiently small strongly pseudoconvex neighbourhood $U$ of $Z$, we have $H^{1}(U, \mathcal{O})=0$ (in particular, each irreducible component of $Z$ must be a rational curve).

The following result is a particular case of [C-L, Main Theorem].

Theorem 4.1 ([C-L]). Let $X, \mathcal{F}, Z=C_{1} \cup \cdots \cup C_{\ell}$ be as above, with $Z$ exceptional and $p_{0}$ rational. Assume that $\left.\operatorname{deg} N_{\mathcal{F}}\right|_{C_{j}}=0$ for every $j=1, \ldots, \ell$. Then there exists a holomorphic function $f$ on some neighbourhood $U$ of $Z$ such that $d f$ generates the foliation on $U$.

Proof: Because $H^{1}(U, \mathcal{O})=0$, the numerical triviality of $N_{\mathcal{F}}$ on $Z$ actually implies that $N_{\mathcal{F}}$ is even holomorphically trivial on $U$, and therefore we may take a holomorphic 1 -form $\omega$ which generates $N_{\mathcal{F}}^{\star}$ over $U$. Thus, we are in the setting of $[\mathbf{C}-\mathbf{L}]$. Namely, by using iteratively the division property (which holds thanks to $H^{1}(U \backslash Z, \mathcal{O})=0$, consequence of $H^{1}(U, \mathcal{O})=0$ and $\left.H_{Z}^{2}(U, \mathcal{O})=0[\mathbf{G}-\mathbf{R}]\right)$, we find formal functions $\hat{f}$ and $\hat{g}$ along $Z$ such that $\omega=\hat{g} d \hat{f}$ (Godbillon-Vey scheme). Then, with some care $[\mathbf{M a l}],[\mathbf{C}-\mathbf{L}]$, we even find that these formal functions can be choosen in a convergent way. 
We now specialize to the following particular case: $Z=C$ is a single smooth rational curve, with normal bundle

$$
N_{C}=\mathcal{O}(-1) \oplus \mathcal{O}(-1) .
$$

It is worth observing that the analytic type of a neighbourhood of such a curve is uniquely determined [Lau]. The singularity $p_{0}$ obtained by collapsing $C$ is isomorphic to the singularity at the origin of the hypersurface in $\mathbb{C}^{4}$ defined by the equation $z_{1} z_{2}=z_{3} z_{4}$, and it is rational.

A fundamental fact for what follows is that the curve $C$ can be flopped.

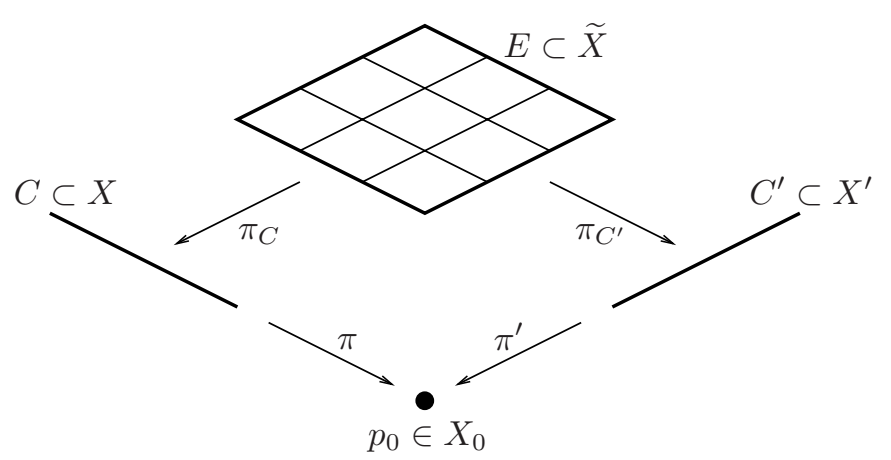

We firstly blow-up $X$ along $C, \pi_{C}: \widetilde{X} \rightarrow X$. The exceptional divisor $E \subset \widetilde{X}$ of $\pi_{C}$ is isomorphic to $\mathbb{C} P^{1} \times \mathbb{C} P^{1}$, and its normal bundle in $\widetilde{X}$ has degree -1 on each factor. On $E$ we have two rulings: the first one is just $E \rightarrow C$, given by the restriction of $\pi_{C}$, and the second one will be denoted by $E \rightarrow C^{\prime}$. Each fiber of this second ruling can be contracted to one point $[\mathbf{F}-\mathbf{N}]$, and the result is a smooth threefold $X^{\prime}$, containing a smooth rational curve $C^{\prime}$ with normal bundle $N_{C^{\prime}}=\mathcal{O}(-1) \oplus \mathcal{O}(-1)$. The space $\widetilde{X}$ can be seen as the blow-up of $X^{\prime}$ along $C^{\prime}, \pi_{C^{\prime}}: \widetilde{X} \rightarrow X^{\prime}$, so that the second ruling above is just the restriction of $\pi_{C^{\prime}}$ to $E$. By collapsing $C^{\prime}$ to one point, we get the same singular threefold $X_{0}$ as by collapsing $C$. Hence, the two collapsing maps

$$
\pi: X \longrightarrow X_{0} \text { and } \pi^{\prime}: X^{\prime} \longrightarrow X_{0}
$$

are two different small resolutions of $p_{0}$, both dominated by the divisorial resolution $\pi \circ \pi_{C}=\pi^{\prime} \circ \pi_{C^{\prime}}: \widetilde{X} \rightarrow X_{0}$.

The bimeromorphic map $\pi_{C^{\prime}} \circ \pi_{C}^{-1}: X \rightarrow X^{\prime}$ (or its inverse) is called a flop of $X$ along $C$ (or of $X^{\prime}$ along $C^{\prime}$ ). 
The foliation $\mathcal{F}$ can be flopped: $\mathcal{F}^{\prime}=\left(\pi_{C^{\prime}}\right)_{*}\left(\pi_{C}\right)^{*}(\mathcal{F})$ is a well defined foliation on $X^{\prime}$. Note, however, that $C^{\prime}$ is not necessarily still a component of $\operatorname{Sing}_{*}\left(\mathcal{F}^{\prime}\right)$ : the foliation $\mathcal{F}^{\prime}$ may have, around $C^{\prime}$, only some isolated singularities on $C^{\prime}$, or even no singularity at all (we shall see later some examples). In fact, it is exactly such a phenomenon that we are looking for, in some sense. The key fact is the following asymmetry between degrees of normal bundles.

\section{Lemma 4.1.}

$$
\left.\operatorname{deg} N_{\mathcal{F}}\right|_{C}=-\left.\operatorname{deg} N_{\mathcal{F}^{\prime}}\right|_{C^{\prime}} .
$$

Proof: Consider the common blow-up $\widetilde{\mathcal{F}}=\pi_{C}^{*}(\mathcal{F})=\pi_{C^{\prime}}^{*}\left(\mathcal{F}^{\prime}\right)$. For general reasons, we have

$$
\begin{aligned}
& N_{\widetilde{\mathcal{F}}}=\pi_{C}^{*}\left(N_{\mathcal{F}}\right) \otimes \mathcal{O}(m E) \\
& N_{\widetilde{\mathcal{F}}}=\pi_{C^{\prime}}^{*}\left(N_{\mathcal{F}^{\prime}}\right) \otimes \mathcal{O}\left(m^{\prime} E\right)
\end{aligned}
$$

for suitable integers $m$ and $m^{\prime}$. Let $F \subset E$ be a fiber of the ruling $E \rightarrow$ $C^{\prime}$, i.e. a section of $E \rightarrow C$, and let us compute the degree of $N_{\widetilde{\mathcal{F}}}$ on $F$, using $E \cdot F=-1$ :

$$
\begin{aligned}
& \left.\operatorname{deg} N_{\widetilde{\mathcal{F}}}\right|_{F}=\left.\operatorname{deg} \pi_{C}^{*}\left(N_{\mathcal{F}}\right)\right|_{F}+m E \cdot F=\left.\operatorname{deg} N_{\mathcal{F}}\right|_{C}-m \\
& \left.\operatorname{deg} N_{\widetilde{\mathcal{F}}}\right|_{F}=\left.\operatorname{deg} \pi_{C^{\prime}}^{*}\left(N_{\mathcal{F}^{\prime}}\right)\right|_{F}+m^{\prime} E \cdot F=-m^{\prime} .
\end{aligned}
$$

By comparison of the two expressions, we get

$$
\left.\operatorname{deg} N_{\mathcal{F}}\right|_{C}=m-m^{\prime} .
$$

By a similar argument, and after exchanging the two rulings of $E$, we get

$$
\left.\operatorname{deg} N_{\mathcal{F}^{\prime}}\right|_{C^{\prime}}=m^{\prime}-m \text {. }
$$

For later purposes, let us observe that the previous lemma holds with no assumptions on the singularities of the foliations, $C$ and $C^{\prime}$ may be totally singular or not.

We can now prove Theorem 1.3.

Let $\mathcal{F}_{0}$ be a foliation on a neighbourhood $U_{0}$ of a simple double point $p_{0} \in X_{0}$, nonsingular on $U_{0}^{\circ}=U_{0} \backslash\left\{p_{0}\right\}$. Under the two above small resolutions, the foliation can be lifted to a neighbourhood $U$ of $C$ and a neighbourhood $U^{\prime}$ of $C^{\prime}$ (for codimensional reasons, for instance, any foliation on $U \backslash C$ extends to $U$ ). Call $\mathcal{F}$ and $\mathcal{F}^{\prime}$ these foliations, and note that

$$
\operatorname{Sing}(\mathcal{F}) \subset C \quad \operatorname{Sing}\left(\mathcal{F}^{\prime}\right) \subset C^{\prime} .
$$


By Lemma 4.1, and up to exchanging the two foliations, we have

$$
\left.\operatorname{deg} N_{\mathcal{F}}\right|_{C} \geq 0
$$

Let us therefore distinguish two cases.

(1) $\left.\operatorname{deg} N_{\mathcal{F}}\right|_{C}=0$. This is the case already analyzed in the above Theorem 4.1 from $[\mathbf{C}-\mathbf{L}]$. It does not matter, for that result, that $C$ be a singular component, the inclusion $\operatorname{Sing}(\mathcal{F}) \subset C$ is enough. Therefore, we are in case (1) of Theorem 1.3.

(2) $\left.\operatorname{deg} N_{\mathcal{F}}\right|_{C}>0$. According to Theorem 1.1, the curve $C$ is not totally singular for $\mathcal{F}$, that is $\operatorname{Sing}(\mathcal{F})$ is only a finite subset of $C$. By [Mal], and up to restricting $U$, we may cover $U$ with open sets $U_{j}$ where the foliation is generated by closed 1-forms $\omega_{j}=d f_{j}, f_{j} \in \mathcal{O}\left(U_{j}\right)$. We claim that the curve $C$ is generically transverse to $\mathcal{F}$. Indeed, in the opposite case $(C$ tangent to $\mathcal{F})$ the Bott vanishing principle would give the flatness of $\left.N_{\mathcal{F}}\right|_{C}$, and therefore the vanishing of its degree, as explained after Proposition 2.2. This generic transversality between $\mathcal{F}$ and $C$ implies, up to restricting $U$, that every leaf of $\mathcal{F}$ (possibly completed with some singular point) is a proper analytic subset of $U$, which intersects $C$ at some points. Hence the leaves of $\mathcal{F}_{0}$ are proper analytic subsets of $U_{0}$ passing through $p_{0}$, and we are in case (2) of Theorem 1.3.

Let us also observe that, in this second case, the (closures of) leaves of $\mathcal{F}^{\prime}$ are also proper analytic subsets of $U^{\prime}$, which however contain the full $C^{\prime}$ instead of intersecting it at a finite set. The foliation $\mathcal{F}^{\prime}$ looks "topologically" like a pencil of hypersurfaces based at $C^{\prime}$.

We conclude with few examples, illustrating case (2).

Example 4.1. As a model of $X$, it is convenient to take the total space of the rank 2 vector bundle $\mathcal{O}(-1) \oplus \mathcal{O}(-1)$, and $C$ equal to the null section. Denote by pr: $X \rightarrow C$ the bundle projection. A first obvious foliation $\mathcal{F}$ is given by the fibers of pr. It is nonsingular, everywhere transverse to $C$, and $\left.\operatorname{deg} N_{\mathcal{F}}\right|_{C}=2$. By flopping, we get a foliation $\mathcal{F}^{\prime}$ with $\operatorname{Sing}\left(\mathcal{F}^{\prime}\right)=C^{\prime}$ and $\left.\operatorname{deg} N_{\mathcal{F}^{\prime}}\right|_{C^{\prime}}=-2$. This foliation is a pencil of hypersurfaces through $C^{\prime}$, which is a so-called Kupka component of radial type: around each point of $C^{\prime}$, the foliation is the product of the radial foliation in dimension $2(z d w-w d z=0)$ and a disc. Both $\mathcal{F}$ and $\mathcal{F}^{\prime}$ have a meromorphic first integral, whose indeterminacy set is either empty or $C^{\prime}$.

The rank 2 vector bundle $\mathcal{O}(-1) \oplus \mathcal{O}(-1)$ has many rank 1 subbundles with trivial quotient (indeed, the dual bundle $\mathcal{O}(1) \oplus \mathcal{O}(1)$ has many trivial rank 1 subbundles, generated by nowhere vanishing sections). 
Every such subbundle

$$
\mathcal{O}(-2) \stackrel{i}{\longrightarrow} \mathcal{O}(-1) \oplus \mathcal{O}(-1) \stackrel{F}{\longrightarrow} \mathcal{O}
$$

induces a submersion

$$
F: X \rightarrow C \times \mathbb{C}
$$

$(C \times \mathbb{C}=$ total space of the trivial line bundle $\mathcal{O})$, whose first component is pr, and the second one is denoted by $f$. If $\mathcal{G}$ is any nonsingular foliation on $C \times \mathbb{C}$, then $\mathcal{F}=F^{*}(\mathcal{G})$ is a nonsingular foliation on $X$. If $\mathcal{G}=\operatorname{Ker}(d f)$, then $\mathcal{F}$ is tangent to $C$, the degree of its normal bundle is 0 , and we are in case (1). Otherwise, $\mathcal{G}$ is generically transverse to $C \times\{0\}$ and $\mathcal{F}$ is generically transverse to $C \subset X$. If $k$ is the number of tangency points (counted with multiplicity), then

$$
\left.\operatorname{deg} N_{\mathcal{F}}\right|_{C}=2+k .
$$

When $k \geq 1$, it is possible that $\mathcal{G}$ admits no meromorphic first integral, on any neighbourhood of $C \times\{0\}$; then also $\mathcal{F}$ admits no meromorphic first integral, on any neighbourhood of $C$. The leaves of the flopped foliation $\mathcal{F}^{\prime}$ are hypersurfaces passing through $C^{\prime}$. At a generic point of $C^{\prime}$ we still have a local product structure, as in the Kupka case, but the 2-dimensional model is more complicated than a radial singularity (singularities of this kind are studied in [Klu]; they also may fail to have a meromorphic first integral). Further, there are some points of $C^{\prime}$ where the local product structure is lost (it is useful to pass through $\widetilde{\mathcal{F}}$ in order to see these properties).

Example 4.2. Take any codimension one foliation $\mathcal{F}_{1}$ in $\mathbb{C} P^{3}$, and take a line $L \subset \mathbb{C} P^{3}$ disjoint from $\operatorname{Sing}_{*}\left(\mathcal{F}_{1}\right)$ and not tangent to $\mathcal{F}_{1}$. Take two points $p_{1}, p_{2} \in L$ where the foliation is transverse to $L$, and blow-up them. Then the strict transform of $L$ is a smooth rational curve $C$ in a threefold $X$ with normal bundle $\mathcal{O}(-1) \oplus \mathcal{O}(-1)$. The lifted foliation $\mathcal{F}$ has at most isolated singularities on a neighbourhood of $C$, and it is generically transverse to $C$. This example is more complicated than the previous one, in the sense that the foliation, around $C$, does not necessarily come from a foliation in dimension 2 . However, we can repeat the considerations above about the structure of the flopped foliation $\mathcal{F}^{\prime}$. Here again we do not have meromorphic first integral around $C$, unless $\mathcal{F}_{1}$ has a rational first integral: indeed, the space of leaves of $\mathcal{F}$ around $C$ is the same as the space of leaves of $\mathcal{F}_{1}$ around $L$, and a meromorphic first integral around $L$ extends to a rational first integral on $\mathbb{C} P^{3}$. 


\section{References}

[B-S] C. BĂNICĂ AND O. STĂNĂŞILĂ, "Méthodes algébriques dans la théorie globale des espaces complexes", Vol. 1, avec une préface de Henri Cartan, Troisième édition, Collection "Varia Mathematica", Gauthier-Villars, Paris, 1977.

[B-B] P. Baum And R. Bott, Singularities of holomorphic foliations, J. Differential Geometry 7 (1972), 279-342.

[Br1] M. BRunella, On the dynamics of codimension one holomorphic foliations with ample normal bundle, Indiana Univ. Math. J. $\mathbf{5 7 ( 7 )}$ (2008), 3101-3113.

[Br2] M. Brunella, Codimension one foliations on complex tori, Ann. Fac. Sci. Toulouse Math. (6) 19(2) (2010), 405-418.

[C-L] D. Cerveau and A. Lins Neto, Frobenius theorem for foliations on singular varieties, Bull. Braz. Math. Soc. (N.S.) 39(3) (2008), 447-469.

[E-V] H. Esnault And E. Viehweg, "Lectures on vanishing theorems", DMV Seminar 20, Birkhäuser Verlag, Basel, 1992.

[F-N] A. Fujiki and S. NaKano, Supplement to "On the inverse of monoidal transformation", Publ. Res. Inst. Math. Sci. 7 (1971/72), 637-644.

[Gra] H. Grauert, Über Modifikationen und exzeptionelle analytische Mengen, Math. Ann. 146 (1962), 331-368.

[G-R] H. Grauert and O. Riemenschneider, Verschwindungssätze für analytische Kohomologiegruppen auf komplexen Räumen, Invent. Math. 11 (1970), 263-292.

[Klu] M. KLUGHERTz, Existence d'une intégrale première méromorphe pour des germes de feuilletages à feuilles fermées du plan complexe, Topology 31(2) (1992), 255-269.

[Lau] H. B. Laufer, On $\mathbb{C} P^{1}$ as an exceptional set, in: "Recent developments in several complex variables" (Proc. Conf., Princeton Univ., Princeton, N. J., 1979), Ann. of Math. Stud. 100, Princeton Univ. Press, Princeton, N.J., 1981, pp. 261-275.

[Lin] A. Lins Neto, A note on projective Levi flats and minimal sets of algebraic foliations, Ann. Inst. Fourier (Grenoble) 49(4) (1999), $1369-1385$.

[Mal] B. Malgrange, Frobenius avec singularités. I. Codimension un, Inst. Hautes Études Sci. Publ. Math. 46 (1976), 163-173.

[Pet] Th. Peternell, Pseudoconvexity, the Levi problem and vanishing theorems, in: "Several complex variables, VII", Encyclopaedia Math. Sci. 74, Springer, Berlin, 1994, pp. 221-257. 
[Rei] M. REID, Bogomolov's theorem $c_{1}^{2} \leq 4 c_{2}$, in: "Proceedings of the International Symposium on Algebraic Geometry" (Kyoto Univ., Kyoto, 1977), Kinokuniya Book Store, Tokyo, 1978, pp. 623-642.

[Suw] T. SuwA, "Indices of vector fields and residues of singular holomorphic foliations", Actualités Mathématiques, Hermann, Paris, 1998.

Marco Brunella:

Institut de Mathématiques de Bourgogne

UMR 5584

9 Avenue Savary

21078 Dijon

France

E-mail address: brunella@u-bourgogne.fr

Carlo Perrone:

Dipartimento di Matematica G. Castelnuovo

Università di Roma La Sapienza

Piazzale A. Moro 5

00185 Roma

Italy

E-mail address: perrone@mat.uniroma1.it

Primera versió rebuda el 25 de gener de 2010,

darrera versió rebuda el 9 de maig de 2011. 